\section{Mortalidade infantil em Cuiabá, Mato Grosso, Brasil, 2005: comparação entre o cálculo direto e após o linkage entre bancos de dados de nascidos vivos e óbitos infantis}

\author{
Infant mortality rate in Cuiabá, Mato Grosso State, \\ Brazil, 2005: a comparison between direct \\ calculation and linkage between the live \\ birth and infant mortality databases
}

Carlos Antônio Maciel de Morais 1

Olga Akiko Takano 1

Jonathan dos Santos Feroldi e Souza 1

\footnotetext{
1 Instituto de Saúde Coletiva, Universidade Federal de Mato Grosso, Cuiabá, Brasil.

Correspondência C. A. M. Morais Universidade Federal de Mato Grosso. Rua Comandante Costa 1701, apto. 123, Cuiabá, MT 78020-400, Brasil. tuke1956@gmail.com
}

\begin{abstract}
This study aimed to analyze the infant mortality rate (IMR) by linking the Live Birth Information System (SINASC) and the Mortality Information System (SIM) and comparing the result to direct calculation using crude data provided by the same databases. The systems used were SINASC, containing 9,590 certificates of live birth from January 1 to December 31, 2005, and SIM, containing 156 death certificates from the reference population (2005). Of the 156 deaths in the year 2005, 126 (80.8\%) were paired by the direct method and 11 (7\%) by manual search, totaling 137 deaths (87.8\%). The rates found with the linkage method allowed calculating the real IMR (14.2 deaths/1,000 live births) and its components. The IMR using linkage was $17.3 \%$ lower than as calculated using crude SIM data. The early neonatal component (7.2 deaths/1,000 live births) accounted for half of the deaths in the first year of life (50.4\%).
\end{abstract}

Information Systems; Infant Mortality Rate; Live Births

\section{Introdução}

O conhecimento dos níveis de mortalidade infantil de uma dada comunidade constitui um elemento precioso para a análise da situação de saúde, auxiliando na avaliação de programas e na vigilância epidemiológica dos agravos à saúde. Também orienta na identificação de grupos populacionais mais expostos ao risco de adoecer e morrer 1.

Apesar da relevância que as informações quantitativas têm para a epidemiologia, sabe-se que nem sempre a informação necessária resulta da simples quantificação dos eventos 2 .

O linkage de banco de dados vem sendo empregado com maior frequência em pesquisas realizadas na área da saúde e consiste na integração de informações de duas bases de dados independentes. Esse método pode ser usado para melhorar a qualidade da informação; para permitir análises de finalidades diferentes daquelas para as quais o banco foi criado, na eliminação de duplicidades de registros, na agregação de informações e para diminuir os custos na aquisição de dados para a pesquisa $3,4,5$.

A técnica de linkage entre o Sistema de Informações sobre Nascidos Vivos (SINASC) e o Sistema de Informações sobre Mortalidade (SIM) torna possível identificar indivíduos ou registros que façam parte de ambos os bancos, possibilitando: (1) o estudo da mortalidade infantil em registros de nascidos vivos de base populacional; 
(2) a estimativa direta da probabilidade de óbito infantil no primeiro ano de vida; (3) o conhecimento de quantos e quais indivíduos de um dado grupo de nascimento evoluiu para o óbito; (4) a estimativa da probabilidade de morte em subgrupos de recém-nascidos, classificados segundo variáveis presentes na Declaração de Nascido Vivo (DNV); (5) o estudo da existência de associação e da magnitude desta entre as variáveis independentes, presentes na DNV e a mortalidade infantil; e (6) o uso de fontes de dados secundárias, disponíveis no SIM e SINASC, para a realização de estudos analíticos longitudinais 6,7,8,9,10,11.

O SINASC e o SIM fazem parte dos Sistemas de Informação em Saúde (SIS) e possibilitam análises comparativas entre os diversos estados, municípios e regiões 5,12,13,14.

O SIM, instituído em 1975, é o mais antigo dos SIS do Brasil e todas as informações relativas aos óbitos são obtidas pelo preenchimento da Declaração de Óbito (DO). A taxa de cobertura do SIM no Brasil exibiu valores que flutuaram entre $80 \%$ e 92\%, entre 1994 e 2004 14,15.

O SINASC, criado em 1990, tem como propósito caracterizar as condições de nascimento. Foi concebido à semelhança do SIM e implantado de forma gradual, desde o ano de 1992. O SINASC recolhe informações pela DNV, padronizada e de distribuição gratuita pelo Ministério da Saúde em todo o país, emitida pelo estabelecimento de saúde onde ocorreu o parto 14,15,16.

Embora seja perceptível que a cobertura do SINASC esteja crescendo e que a qualidade da informação venha melhorando desde a sua implantação, sabe-se que a taxa de cobertura do sistema ainda não é completa, particularmente nas regiões Norte e Nordeste 14,17.

O conhecimento da qualidade da informação do SINASC e do SIM, tanto do ponto de vista qualitativo, que implica a fidedignidade das informações, quanto quantitativo, que analisa a cobertura total dos eventos e a completude do preenchimento, é fundamental para o conhecimento da potencialidade epidemiológica desse sistema 14,17,18.

A aplicação da técnica de linkage, em estudos sobre mortalidade no Brasil, só se tornou possível com a melhora na completude e na confiabilidade das informações prestadas pelo SINASC e pelo SIM. Diversos estudos classificados como epidemiológicos foram realizados, com predominância do desenho de coorte, com uso de arquivos do SIM/SINASC, tendo como tema mortalidade infantil, especialmente a neonatal 3,6,7,8,9,16,19,20,21,22.

Este estudo foi realizado no Município de Cuiabá, Mato Grosso - 526.830 habitantes (IBGE; http://www.ibge.gov.br/cidadesat/topwindow. htm?1, acessado em 27/Mar/2008) - localizado na Região Centro-oeste do Brasil. Cuiabá, capital do estado, foi o primeiro município de Mato Grosso, criado por carta régia, em 8 de abril de 1719.

O estudo teve como objetivo determinar as Taxas de Mortalidade Infantil (TMI) e seus componentes no Município de Cuiabá, no ano de 2005, utilizando-se o método direto, mediante dados brutos de nascimentos e de óbitos, retirados dos bancos de dados do SINASC e SIM; compará-las com a encontrada pela utilização da técnica de linkage.

\section{Metodologia}

Estudo observacional, de corte transversal, de base populacional, utilizando base de dados secundários de nascimentos e óbitos de crianças menores de um ano.

Foi considerada como população de estudo o conjunto de nascidos vivos ocorridos em mães residentes no Município de Cuiabá, no período de 1 o de janeiro de 2005 a 31 de dezembro de 2005. Fazem parte também deste estudo os óbitos com menos de um ano de vida, originados dos nascidos vivos em estudo, compreendendo as mortes ocorridas entre 1o de janeiro de 2005 a 31 de dezembro de 2006, em Cuiabá ou em outros municípios.

O método de cálculo das taxas de mortalidade infantil, empregado neste estudo e referido no texto como "direto" foi aquele que utilizou os dados brutos de nascimentos e de óbitos, retirados dos bancos de dados do SINASC e SIM, sem os ajustes empregados no método de linkage.

$\mathrm{O}$ encadeamento de arquivos (linkage) é a integração de informações de duas bases de dados independentes, buscando a correção da informação quando se acredita na validade dela em um dos bancos ou adicionar informações de um banco de dados para outro. No encadeamento, os registros de cada uma das fontes, tidos como relacionados a um mesmo indivíduo, são pareados (matched) de forma a poder tratá-los como um registro único para aquele indivíduo. O pareamento é empregado para se juntar registros correspondentes ao mesmo indivíduo de dois ou mais arquivos ou duplicatas de registros dentro de um mesmo arquivo. O encadeamento de arquivos por pareamento pode permitir uma interoperabilidade (interconexão efetiva) entre sistemas de informações 3,4,5,11,23,24,25.

Na técnica de linkage, utilizou-se no processo de blocagem (blocking) uma chave formada pela combinação do código soundex, que é um código fonético frequentemente usado para este fim do 
primeiro nome, buscando a combinação exata, além do sexo e da data de nascimento. Como o código soundex retém a primeira letra do nome, as diferentes grafias recebem códigos diferentes, o que aumenta a probabilidade de perda de pares verdadeiros 11,23,24.

Cada DO foi emparelhada com a respectiva DNV, de acordo com os seguintes procedimentos: (1) uma pesquisa automática no banco de dados de nascidos vivos no Município de Cuiabá, utilizando campos comuns à DNV e à DO (data de nascimento, sexo e nome da mãe) para selecionar o conjunto de DNV-DO que apresentavam a mesma data de nascimento, sexo e nome da mãe; (2) pesquisa manual utilizando fragmentos do nome da mãe na DO buscando localizá-la na DNV, fazendo pareamento com a data de nascimento e sexo, para os casos não detectados na pesquisa automática; (3) pesquisa automática no banco de dados de nascidos vivos em outros municípios do estado, utilizando campos comuns à DNV e à DO (data de nascimento, sexo e nome da mãe) para selecionar o conjunto de DNV-DO que apresentavam a mesma data de nascimento, sexo e nome da mãe; (4) pesquisa manual nos arquivos de DNV objetivando confirmar a(s) DNV(s) identificada(s) no procedimento de busca automática utilizando a variável nome da mãe, sexo e data de nascimento.

A "Probabilidade M", que indica a probabilidade dos valores das variáveis de pareamento (matching) concordar, dado que o par é referente à mesma pessoa, para o nome da mãe foi de 0,96 , para a data de nascimento foi de 0,95 e para o sexo foi também de 0,95 ; todos buscando uma combinação exata dos dados durante o pareamento 11,23.

Em razão da inclusão da revisão manual no processo, foi utilizado o ponto de corte (cut off value) de quatro, buscando conseguir um maior número de pareamentos. $\mathrm{O}$ escore que mede o grau de concordância entre os pareamentos encontrados variou de 9,3 a 7,0 23 .

Foram utilizados os bancos de dados do SIM e do SINASC fornecidos pelo setor responsável pela sua operacionalização, processamento e produção dos dados dentro da Secretaria de Estado de Saúde de Mato Grosso (SES/MT): a Coordenadoria de Vigilância Epidemiológica, setor subordinado à Superintendência de Saúde Coletiva-SES/MT.

Os dados originais, em arquivos TabWin do Departamento de Informática do SUS (DATASUS; http://www.datasus.gov.br), foram fornecidos pela SES/MT e armazenados em planilhas do Microsoft Excel 2007 (Microsoft Corp., Estados Unidos). Os cálculos foram realizados em Epi Info versão 3.3.2 (Centers for Disease Control and
Prevention, Atlanta, Estados Unidos) e o procedimento de linkage foram realizados no programa Link Plus versão 1.0 (Centers of Disease Control and Prevention, Atlanta, Estados Unidos).

Para a identificação dos óbitos infantis ocorridos no período, foi utilizada a técnica de linkage entre os bancos de dados do SINASC e do SIM. Com base nos registros de nascidos vivos, no Município de Cuiabá, no ano 2005, foi feito o pareamento, visando à unificação dos bancos de dados, entre as DNV e as respectivas DO relativas aos óbitos infantis dos registro em estudo.

Durante o encadeamento de base de dados entre DNV e DO, foi realizada busca manual com objetivo de minimizar erros neste estudo, como a duplicidade dos registros, erros de preenchimento de campos vitais como: nome da mãe, sexo, data nascimento, data do óbito, raça/cor. As informações contidas nas DNV foram consideradas como padrão ouro quando surgiam divergências ou ausências de informação nas DO.

Foi realizada a revisão manual de todos os pares encontrados para minimizar tal viés. A data de nascimento foi de importância fundamental na inclusão da chave, sobretudo nos estudos mais longos, quando existe a possibilidade de mais de um nascimento no período estudado.

O método de pareamento DNV-DO direto utilizou o método padrão do programa Link Plus versão 1.0 para extrair as probabilidades "M" usadas no processo de pareamento dos registros.

O presente trabalho foi submetido ao Comitê de Ética do Hospital Universitário Júlio Müller (HUJM), sob o protocolo CEP/HUJM no. 375/07, em 8 de agosto de 2007, e aprovado.

\section{Resultados}

A Figura 1 mostra o modelo explicativo do processo de linkage utilizado para o cálculo da casuística final deste estudo.

Inicialmente, foi montado o banco de dados dos nascidos vivos de mães residentes no Município de Cuiabá em 2005, um total de 9.590 nascidos vivos e de óbitos em 2005, com 166 óbitos.

Paralelamente, foi analisado o banco de dados SIM 2005 e 2006. Foram encontrados e excluídos 24 óbitos relacionados em 2005 pertencentes a nascidos vivos em 2004 e incluídos 14 óbitos relacionados em 2006 por pertencerem a nascidos vivos em 2005. Após essa análise, permaneceu no banco de dados um total de 156 óbitos.

Pelo processo de "linkage", foram encontrados 126 óbitos por pareamento direto, sendo todos revisados manualmente e comparados às respectivas DNV. Em decorrência da imprecisão no preenchimento do campo "nome da mãe", foi 
Modelo explicativo do procedimento de linkage entre os bancos de dados do Sistema de Informações sobre Nascidos Vivos (SINASC) 2005 e do Sistema de Informações sobre Mortalidade (SIM) 2005 e 2006. Cuiabá, Mato Grosso, Brasil.

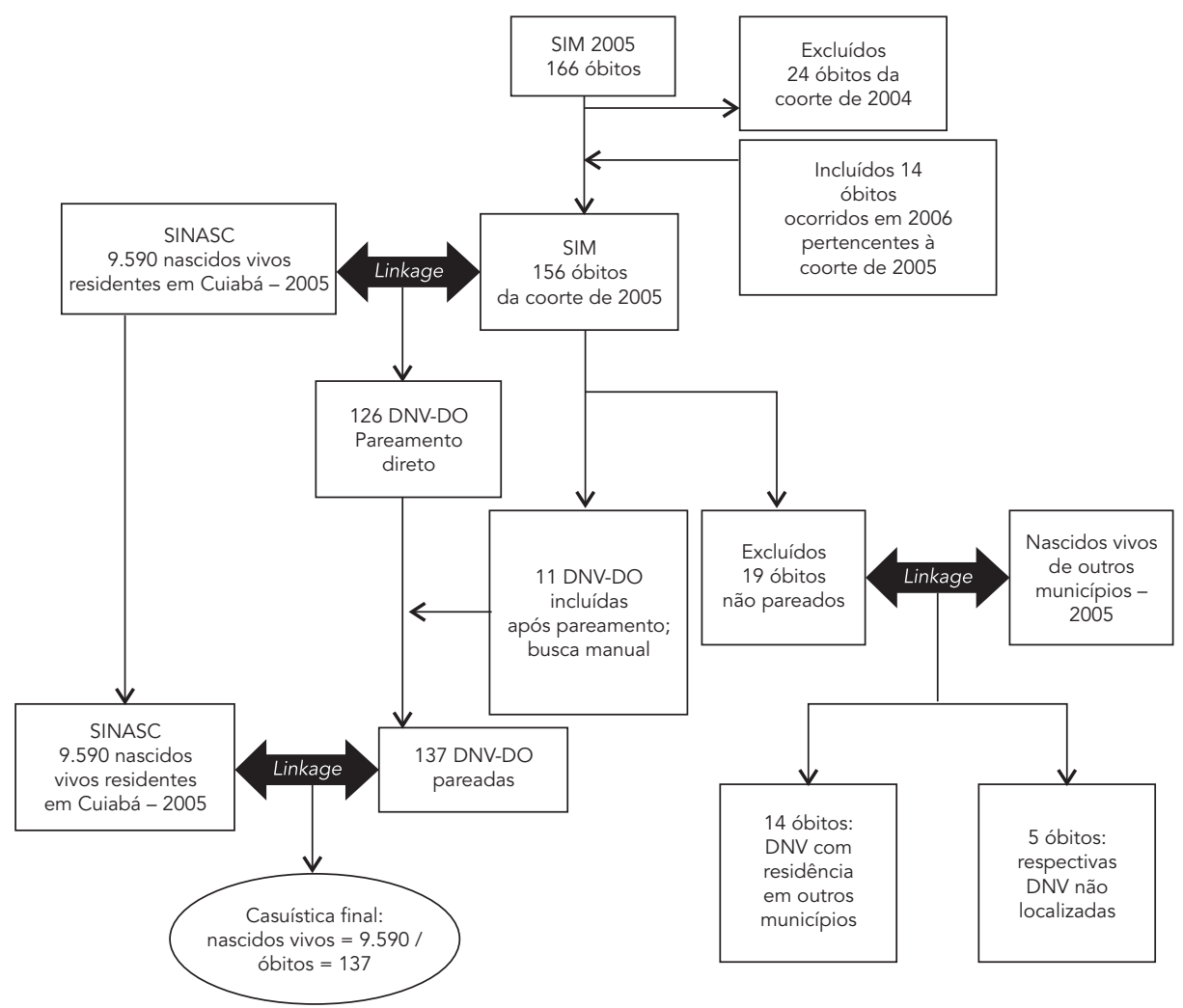

DNV: Declaração de Nascido Vivo; DO: Declaração de Óbito.

realizado busca manual para as DO não pareadas usando fragmentos do nome da mãe nas DO e realizando a busca no banco de dados das DNV. Foram encontrados 11 pareamentos indiretos, sendo conferidos outros dados como sexo, data de nascimento, além da similaridade na grafia do nome materno. No total foram pareados 137 DNV-DO (Figura 1).

Dessa forma, através do método de linkage, a casuística final foi de 137 óbitos, num total de $87,8 \%$ do total inicial dos óbitos encontrados (156), sendo $126(80,8 \%)$ pareados pelo método direto e 11 (7\%) pelo método de busca manual.

Foram excluídos 19 óbitos (12,2\%) cujo pareamento não foi encontrado pelo processo de linkage com as DNV ocorridas no Município de Cuiabá, em 2005. Foi realizado um novo linkage entre os 19 óbitos não pareados com as DNV ocorridas em outros municípios do Estado de Mato Grosso, em 2005. Foram encontrados 14 pareamentos DNV-DO, isto é, 14 óbitos (9\%) pertenciam a outros municípios do estado, restando apenas 5 óbitos $(3,2 \%)$ não pareados com nenhuma DN de Cuiabá ou de outro município de Mato Grosso.

Os dados de óbitos obtidos pelo método direto e de linkage encontram-se na Tabela 1.

A TMI de seus componentes calculada pelo método direto e utilizando o método de linkage encontra-se na Tabela 2.

\section{Discussão}

A subnotificação de eventos, um percentual excessivo de causas mal definidas de óbito, erros de classificação de óbitos fetais e não fetais, além do preenchimento incorreto ou incompleto da DNV e DO resultam em variações na qualidade do SIM/SINASC e modificam os estudos que 
Distribuição dos óbitos ocorridos (N) no primeiro ano de vida de 9.590 nascidos vivos em Cuiabá, Mato Grosso, Brasil, no ano de 2005, segundo dados brutos do Sistema de Informações sobre Mortalidade (SIM) e após procedimento de linkage.

\begin{tabular}{lcc}
\hline Óbitos & Dados do SIM (N) & Dados após linkage (N) \\
\hline Óbito neonatal & 100 & 88 \\
Neonatal precoce & 77 & 69 \\
Neonatal tardia & 23 & 19 \\
Óbito pós-neonatal & 66 & 49 \\
Óbito infantil & 166 & 137 \\
\hline
\end{tabular}

Fonte: Departamento de Informática do SUS/Secretaria de Estado de Saúde de Mato Grosso/Sistema de Informações sobre Nascidos Vivos/Sistema de Informações sobre Mortalidade.

Tabela 2

Distribuição da Taxa de Mortalidade Infantil e seus componentes, segundo dados do Sistema de Informações sobre Nascidos Vivos (SINASC)/Sistema de Informações sobre Mortalidade (SIM) e após o procedimento de linkage. Cuiabá, Mato Grosso, Brasil, 2005

\begin{tabular}{lccc}
\hline Taxa de mortalidade & Dados do SIM/SINASC & Dados após linkage & Variação (\%) \\
\hline Infantil & 17,3 & 14,3 & $-17,3$ \\
Neonatal precoce & 8,0 & 7,2 & $-10,0$ \\
Neonatal tardia & 2,4 & 2,0 & $-16,7$ \\
Pós-neonatal & 6,9 & 5,1 & $-26,1$ \\
\hline
\end{tabular}

Fonte: Departamento de Informática do SUS/Secretaria de Estado de Saúde de Mato Grosso/Sistema de Informações sobre Nascidos Vivos/Sistema de Informações sobre Mortalidade.

tomam como base estes bancos de dados. Os óbitos neonatais, notificados como óbitos fetais, refletem que ainda é comum em nosso meio ignorar óbitos de crianças muito prematuras e/ou ocorridos logo após o nascimento, o que implica a notificação incorreta como óbito fetal pela ausência da emissão da DNV, conforme apontado em alguns trabalhos 3,14,19,20.

Algumas medidas podem ser tomadas na minimização dessas falhas. O melhor preparo técnico das pessoas responsáveis pelas notificações; o uso de mecanismos como o linkage na construção de um banco de dados mais completos e com um menor viés de informação forneceria, por intermédio do encadeamento de bancos de dados de nascimentos e óbitos, informações sobre quantos e quais indivíduos do banco de dados estudado evoluem para o óbito. O linkage permite uma maior completude das informações e possibilitou a recuperação de informações além de eliminar duplicidades dentro de um mesmo arquivo 11,17,22,23,24,25.
Os ajustes realizados na construção do banco de dados dos óbitos indicaram a ocorrência de possíveis falhas de inclusão de registros no SINASC. Neste estudo, observou-se que 11 óbitos (7\%) foram encontrados apenas pela busca manual, revelando falhas tanto no preenchimento correto das DNV como também das DO. A exclusão de 19 óbitos do estudo (12,2\%) evidenciou também falhas na emissão das DNV, pois 14 deles (9\%) pertenciam a outros municípios do estado. Em apenas 5 óbitos (3,2\%) não se conseguiu o pareamento por conta da ausência das respectivas DNV.

A impossibilidade de emparelhamento DNVDO, por possível falha na emissão das DNV, tem sido encontrada em outros estudos, variando de $40 \%$ no Estado do Rio de Janeiro para os registros de óbitos em menores de um ano analisados 24; 27,5\% para os óbitos neonatais precoces em Salvador, Bahia 21; 9\% nos estudos realizados em Santo André, São Paulo, para os óbitos neonatais 3,26,27; $5 \%$ dos óbitos neonatais, com baixo peso 
ao nascimento em Recife, Pernambuco 10; 3,8\% dos óbitos em menores de um ano no Distrito Federal 28 e $2 \%$ dos óbitos nesta mesma faixa etária em Goiânia, Goiás 6.

A técnica de encadeamento ou linkage de banco de dados possibilitou a unificação de duas bases de dados, SIM e SINASC, mediante as informações comuns registradas, permitindo a identificação de um mesmo indivíduo e suas características, presentes em ambos os bancos.

As dificuldades encontradas no procedimento de linkage dos bancos de dados SINASC e SIM foram referentes às divergências encontradas no campo "nome da mãe" nas DNV e nas DO, requerendo a confirmação por outros campos como: idade, sexo, data de nascimento para confirmar o pareamento.

Verificou-se que o uso da técnica de linkage permitiu mostrar a real taxa de mortalidade na população estudada e que as taxas obtidas pelo método direto estão superestimadas. A TMI utilizando o método de linkage foi $17,3 \%$ menor que a obtida pelo uso do número de registro do SIM pelo método direto. Se essa TMI encontrada pelo método de linkage fosse utilizada na tabela de ranqueamento da mortalidade infantil entre as capitais, Cuiabá passaria do 15o para o 10o lugar (DATASUS. http://tabnet.datasus.gov.br/cgi/ tabcgi.exe?sim/cnv/infbr.def, acessado em 10/ Ago/2009).

Todos os componentes da TMI sofreram redução com a aplicação do linkage, porém mantendo a mesma distribuição das freqüências, com predominância da Taxa de Mortalidade Infantil Neonatal Precoce (TMINP).

Nos dois métodos de cálculos utilizados, encontrou-se um predomínio da Taxa de Mortalidade Infantil Neonatal (TMIN), e a TMINP foi predominante em ambos os métodos utilizados.

Em anos recentes, a taxa de mortalidade infantil tem caído especialmente por causa da redução da mortalidade pós-neonatal, reflexo da melhoria da atenção básica à criança e dos fatores associados ao meio ambiente (sobretu- do água e saneamento). Dessa forma, tornou-se proporcionalmente maior a participação dos outros dois componentes neonatais na taxa de mortalidade. Eles, para serem atenuados, dependem do aperfeiçoamento do atendimento à mãe desde a gestação e o parto até o pós-parto, bem como da qualidade da atenção ao recém-nascido com condições especiais de risco 29,30.

A maior predominância dos óbitos infantis no período neonatal neste estudo, particularmente no período neonatal precoce, foi observada também em outros estudos 16,29,30. Tais achados sugerem que as causas de mortalidade no primeiro ano de vida verificadas neste estudo, podem estar associadas especialmente com a qualidade e condições de assistência à gestação e ao parto. Demonstra a necessidade de uma maior articulação dos serviços de atenção básica e assistência ao parto, dependendo de estratégias no campo perinatal, com ações dirigidas ao desenvolvimento e fortalecimento dos serviços de saúde materno-infantil 30,31,32.

Esta técnica permite aos gestores públicos uma melhor qualidade das informações dos óbitos e sobre o impacto dos óbitos de outras procedências na composição da TMI do município, permitindo melhor planejamento de políticas públicas para a redução da mortalidade infantil.

Por causa de seu baixo custo operacional e facilidade de emprego, o uso dessa técnica na construção e recuperação de dados permitirá a construção de bases de dados com informações mais confiáveis e de melhor completude. Facilitará estudos de planejamento e avaliação da saúde materno-infantil, além do monitoramento da prevalência dos fatores de risco e de sua magnitude na população de nascidos vivos.

Apesar das restrições metodológicas, inerentes ao uso de dados secundários, este procedimento mostrou-se útil na estimação da TMI real, no Município de Cuiabá, no ano de 2005 e aponta necessidade de investigar as causas evitáveis de mortalidade neonatal precoce, principal causa de óbito no primeiro ano de vida no município. 


\section{Resumo}

O objetivo deste estudo foi avaliar a Taxa de Mortalidade Infantil (TMI) pela utilização do método de linkage entre o banco de dados do Sistema de Informação sobre Nascidos Vivos (SINASC) e sobre mortalidade (SIM) e compará-la com a encontrada nos dados brutos fornecidos pelas mesmas bases de dados. Foi utilizado o SINASC contendo 9.590 Declarações de Nascidos Vivos (DNV) entre 1o de janeiro e 31 de dezembro de 2005 e o SIM com 156 Declarações de Óbitos (DO) relativas à população estudada. Dos 156 óbitos relativos ao ano de 2005 foram pareados pelo método direto 126 (80,8\%) e pelo método de busca manual 11 (7\%), totalizando um total de 137 óbitos (87,8\%). As taxas encontradas com o método de linkage permitiram estimar a real TMI (14,2 óbitos/1.000 nascidos vivos) e a de seus componentes. A TMI encontrada pelo uso do método de linkage foi 17,3\% menor que a calculada peça utilização dos registros brutos do SIM, permanecendo o componente neonatal precoce (7,2 óbitos/1.000 nascidos vivos) como o principal responsável pelos óbitos no primeiro ano de vida (50,4\%).

Sistemas de Informação; Coeficiente de Mortalidade Infantil; Nascidos Vivos

\section{Colaboradores}

Todos os autores participaram na elaboração do texto, concepção do artigo, metodologia, análise, elaboração das tabelas e figuras.

\section{Agradecimentos}

À Fundação de Apoio à Pesquisa do Estado de Mato Grosso (FAPEMAT; edital PPSUS-MT 2006/FAPEMAT no. 010/2006, de "apoio a projetos de pesquisa para o SUS").

\section{Referências}

1. Mota E, Carvalho DMT. Sistemas de informação em saúde. In: Rouquayrol MZ, Almeida Filho N, organizadores. Epidemiologia \& saúde. 6a Ed. Rio de Janeiro: Medsi; 2003. p. 605-28.

2. Silva JPL, Travassos C, Vasconcellos MM, Campos LM. Revisão sistemática sobre encadeamento ou linkage de base de dados secundários para uso em pesquisa em saúde no Brasil. Cad Saúde Colet (Rio J.) 2006; 14:197-224.

3. Almeida MF, Mello-Jorge MHP. O uso da técnica de "Linkage" de sistemas de informação em estudos de coorte sobre mortalidade neonatal. Rev Saúde Pública 1996; 30:141-7.

4. Wincler WE. Machine learning, information retrieval, and record linkage. U.S. Bureau of the Census Statistical Research. http://www.niss.org/ affiliates/dqworkshop/papers/winkler.pdf (acessado em 17/Jun/2009).

5. Pinheiro RS, Camargo Jr. KR, Coeli CM. Relacionamento de bases de dados em saúde. Cad Saúde Colet (Rio J.) 2006; 14:195-6.
6. Morais Neto OL, Barros MBA. Fatores de risco para mortalidade neonatal e pós-neonatal na Região Centro-Oeste do Brasil: linkage entre bancos de dados de nascidos vivos e óbitos infantis. Cad Saúde Pública 2000; 16:477-85.

7. Martins EF, Velásquez-Meléndez G. Determinantes da mortalidade neonatal a partir de uma coorte de nascidos vivos, Montes Claros, Minas Gerais, 1997-1999. Rev Bras Saúde Matern Infant 2004; 4 : 405-12.

8. Santa Helena ET, Sousa CA, Silva CA. Fatores de risco para mortalidade neonatal em Blumenau, Santa Catarina: linkage entre banco de dados. Rev Bras Saúde Matern Infant 2005; 5:209-17.

9. Silva CF, Leite AJM, Almeida NMGS, Gondim RC. Fatores de risco para mortalidade infantil em município do Nordeste do Brasil: linkage entre banco de dados de nascidos vivos e óbitos infantis - 2000 a 2002. Rev Bras Epidemiol 2006; 9:69-80. 
10. Ribeiro AM, Guimarães MJ, Lima MC, Sarinho SW. Fatores de risco para mortalidade neonatal em crianças com baixo peso ao nascer. Rev Saúde Pública 2009; 43:246-55.

11. Machado JP, Silveira DP, Santos IS, Piovesan MF, Albuquerque C. Aplicação da metodologia de relacionamento probabilístico de base de dados para a identificação de óbitos em estudos epidemiológicos. Rev Bras Epidemiol 2008; 11:43-54.

12. Pedrosa LDCO, Sarinho SW, Ordonha MAR. Óbitos neonatais: por que e como informar. Rev Bras Saúde Matern Infant 2005; 5:411-8.

13. Ministério da Saúde. Sistemas de Informação sobre Mortalidade (SIM) e Nascidos Vivos (SINASC) para os profissionais do Programa Saúde da Família. 2a Ed. Brasília: Ministério da Saúde; 2004.

14. Frias GF, Pereira PMH, Andrade CLT, Szwarcwald CL. Sistema de Informação sobre Mortalidade: estudo de caso em municípios com precariedade de dados. Cad Saúde Pública 2008; 24:2257-66.

15. Secretaria de Vigilância em Saúde, Ministério da Saúde. Sistema nacional de vigilância em saúde: relatório de situação: Mato Grosso/MS/SVS. Brasília: Ministério da Saúde; 2006

16. Sarinho SW, Coutinho SB, Acioli TML. Mortalidade neonatal em Recife, Pernambuco, 1998: causas básicas e grau de conhecimento dos neonatologistas acerca do preenchimento das Declarações de Óbito. J Pediatr (Rio J.) 2001; 23:279-84.

17. Szwarcwald CL, Leal MC, Andrade CLT, Souza Jr. PRB. Estimação da mortalidade infantil no Brasil: o que dizem as informações sobre óbitos e nascimentos do Ministério da Saúde. Cad Saúde Pública 2002; 18:1725-36.

18. Romero DE, Cunha CB. Avaliação da qualidade das variáveis epidemiológicas e demográficas do Sistema de Informação de Nascidos Vivos, 2002. Cad Saúde Pública 2007; 23:701-14.

19. Nascimento EMR, Costa MCN, Mota ELA, Paim JS. Estudos de fatores de risco para óbitos de menores de um ano mediante compartilhamento de banco de dados. Cad Saúde Pública 2008; 24:2593-602.

20. Silva CF, Leite AJM, Almeida NMGS. Linkage entre banco de dados de nascidos vivos e óbitos infantis em município do Nordeste do Brasil: qualidade dos sistemas de informação. Cad Saúde Pública 2009; 25:1552-8

21. Soares ES, Menezes GMS. Fatores associados à mortalidade neonatal precoce: análise de situação no nível local. Epidemiol Serv Saúde 2010; 19: 51-60.

22. Pereira PMH, Frias PG, Carvalho PI, Vidal AS. Mortalidade neonatal na coorte de nascidos vivos em maternidade-escola na Região Nordeste do Brasil, 2001-2003. Epidemiol Serv Saúde 2006; 15:19-28.
23. Coeli CM, Camargo Jr. KR. Avaliação de diferentes estratégias de blocagem no relacionamento probabilístico de registros. Rev Bras Epidemiol 2002; 5:185-96.

24. Reis AC. Recuperação de dados da Declaração de Óbitos (DO) para menores através da Declaração de Nascidos Vivos (DN). Cad Saúde Colet (Rio J.) 2006; 14:297-304.

25. Pereira APE, Gama SGN, Leal MC. Mortalidade infantil em uma amostra de nascimentos do município do Rio de Janeiro, 199-2001: "linkage" com o Sistema de Informação de Mortalidade. Rev Bras Saúde Matern Infant 2007; 7:83-8.

26. Almeida MF. Mortalidade neonatal em Santo André [Tese de Doutorado]. São Paulo: Faculdade de Saúde Pública, Universidade de São Paulo; 1994.

27. Almeida MF, Mello-Jorge MHP. O uso da técnica de linkage de sistemas de informação em estudos de coorte sobre mortalidade neonatal. Rev Saúde Pública 1996; 30:141-7.

28. Fernandes DM. Concatenamento de informação sobre óbitos e nascimentos: uma experiência me todológica do Distrito Federal 1986-1991 [Tese de Doutorado]. Belo Horizonte: Centro de Desenvolvimento e Planejamento Regional, Universidade Federal de Minas Gerais; 1997.

29. Departamento de Análise de Situação de Saúde, Secretaria de Vigilância em Saúde, Ministério da Saúde. Saúde Brasil 2008: 20 anos de Sistema Único de Saúde (SUS) no Brasil. Brasília: Ministério da Saúde; 2009.

30. Instituto de Pesquisa Econômica Aplicada/Secretaria de Planejamento e Investimentos Estratégicos, Ministério do Planejamento. Objetivos de desenvolvimento do milênio: relatório nacional de acompanhamento. Brasília: Instituto de Pesquisa Econômica Aplicada/Secretaria de Planejamento e Investimentos Estratégicos, Ministério do Planejamento; 2007.

31. Campos D, Loschi RH, França E. Mortalidade neonatal precoce hospitalar em Minas Gerais: associação com variáveis assistenciais e a questão da subnotificação. Rev Bras Epidemiol 2007; 10:223-38

32. Drumond EF, Machado CJ, França E. Óbitos neonatais precoces: análise de causas múltiplas de morte pelo método Grade of Membership. Cad Saúde Pública 2007; 23:157-66.

Recebido em 11/Abr/2010

Versão final reapresentada em 04/Out/2010

Aprovado em 29/Nov/2010 